\title{
Invasive pneumococcal disease in New South Wales, Australia: reporting Aboriginal and Torres Strait Islander status improves epidemiology
}

\author{
Peter D Massey, ${ }^{a}$ Kerry Todd, ${ }^{a}$ Maggi Osbourn, ${ }^{,}$Kylie Taylor ${ }^{a}$ and David N Durrheim ${ }^{a}$ \\ Correspondence to Peter D Massey (e-mail: Peter.Massey@hnehealth.nsw.gov.au).
}

The aim of this work was to determine the feasibility of improving Aboriginal and Torres Strait Islander status recording for notifiable diseases using all Invasive Pneumococcal Disease (IPD) notifications in a regional area of New South Wales, Australia.

In Australia people with IPD are nearly always admitted to hospital and their Aboriginal and Torres Strait Islander status is recorded. Aboriginal and Torres Strait Islander status was determined for IPD notifications by referring to the routine hospital admission data in a regional area of New South Wales, Australia.

There were 234 notifications in the regional area of Hunter New England during the period 2007-2009. Initially, 168 (72\%) notifications had Aboriginal and Torres Strait Islander status recorded. After referring to the routine hospital admission data, the recorded status increased to 232 (99\%). Updating the surveillance data required less than five minutes per notification.

Referring to routine hospital admission data proved a useful and time-efficient surveillance strategy to increase the proportion of notifications with Aboriginal and Torres Strait Islander status. These data can then be used to better understand the current epidemiology of IPD. Aboriginal and Torres Strait Islander children aged 0-4 years have a two- to threefold higher rate of invasive pneumococcal disease than non-Aboriginal children, thus high levels of timely pneumococcal immunization coverage remain important for young Aboriginal and Torres Strait Islander children.

nvasive Pneumococcal Disease, caused by Streptococcus pneumoniae, can result in pneumonia, meningitis, sinusitis and otitis media. Less frequently this gram-positive encapsulated coccus causes endocarditis, septic arthritis and peritonitis. ${ }^{1,2}$ For the purpose of notification, a case of IPD is defined as: "the isolation from or the detection by nucleic acid test of $S$. pneumoniae in blood, cerebrospinal fluid or other sterile site." ${ }^{3}$ IPD has been notifiable by laboratories in New South Wales (NSW), Australia, since December 2000 under the NSW Public Health Act 2010. Case information is entered into the NSW Notifiable Conditions Information Management System by Public Health Units. Collection of enhanced surveillance data in NSW includes Aboriginal and Torres Strait Islander status for notified cases $0-5$ years of age and 50 years and older. In Australia people with IPD are nearly always admitted to hospital and their Aboriginal and Torres Strait Islander status is recorded.
Enhanced surveillance for notifications of IPD also includes risk factors and vaccination history. The enhanced surveillance commenced in NSW during 2002 following the introduction of a publicly funded 7-valent conjugate vaccine for Aboriginal and Torres Strait Islander children and a publicly funded 23-valent vaccine for Aboriginal and Torres Strait Islander adults 50 years and over in 1999. Aboriginal and Torres Strait Islander people aged 15 years and older with a chronic condition are also eligible for the publicly funded 23 -valent vaccine.

The risk factors associated for IPD include prematurity (less than 37 weeks gestation), congenital or chromosomal abnormality, anatomical or functional asplenia, immunocompromised status, chronic illness, childcare attendee, previous episode of IPD, and other (for example tobacco use). ${ }^{3}$ Several of these risk factors are more prevalent in Aboriginal and Torres Strait Islander people. ${ }^{4}$ Data on Aboriginal and Torres Strait Islander

\footnotetext{
a Hunter New England Population Health, Tamworth, Australia 
status, vaccination history and risk factors are collected during enhanced surveillance of the disease.

A recent study found that, despite the introduction of a publicly funded vaccination programme in Australia, the IPD burden continues to disproportionately affect Aboriginal and Torres Strait Islander people, including young adults., ${ }^{3,5}$ The Australian Aboriginal and Torres Strait Islander Health/nfoNet reported in 2009 that in selected states/territories the incidence of IPD among Aboriginal and Torres Strait Islander people aged 25-49 years was 11.2 times higher (50.9 per 100 000) than that among non-Aboriginal people (4.5 per 100 000). ${ }^{5}$ The high rates of IPD notifications among Aboriginal and Torres Strait Islander people in Australia are also reflected in hospitalization rates for pneumococcal septicaemia and meningitis. ${ }^{6}$

Enhanced surveillance (including Aboriginal and Torres Strait Islander status) for IPD in all ages is collected and reported for notifications in Northern Territory, most of Queensland, Tasmania, South Australia, Victoria and Western Australia. ${ }^{3}$ NSW notification data do not currently routinely include Aboriginal and Torres Strait Islander status for people aged 5-49 years of age, thus it is not known what the burden of the disease is in Aboriginal and Torres Strait Islander people in NSW in that age group.

\section{METHOD}

Aboriginal and Torres Strait Islander status was determined for IPD notifications during the period 2007-2009 in the regional area of Hunter New England (HNE) in northern NSW by referring to their routine hospital admission data. Routine hospital admission data in Australia includes demographics, presentation and discharge dates, discharge diagnosis codes and outcome data. Public health clinicians in this regional area have access to the Clinical Applications Portal database which is an electronic demographic and clinical information system within the Health Service. Notified IPD cases were checked against the admission data for the relevant admission using name, date of birth, country of birth, language spoken at home and date of admission. The Aboriginal and Torres Strait Islander status from routine hospital admission data were updated into the notifiable conditions database. The public health time and resources required to conduct this data checking were also recorded.

IPD notification data for the period 2007-2009 for the regional area were sourced from the Health
Outcomes Information and Statistical Toolkit, NSW Department of Health. Analysis was performed using Microsoft Excel 2003, with notification rates calculated using mid-term estimate population figures from the Australian Bureau of Statistics 2006 Census and 2009 estimates as denominators.

The recording of Aboriginal and/or Torres Strait Islander status in the notifiable conditions database was assessed as complete if a valid response ("yes" or "no") was recorded in the Aboriginal and/or Torres Strait Islander field.

Three-year mean IPD notification rates were then determined for Aboriginal and Torres Strait Islander people and the non-Aboriginal population to allow calculation of a relative risk of IPD notification. Direct age-standardization was used to control for the relatively younger Aboriginal and Torres Strait Islander population, using the non-Aboriginal population in HNE as the standard.

This project was deemed a quality improvement exercise by the Hunter New England Human Research Ethics Committee and so did not need ethics approval. One member of the team, an Aboriginal person, was responsible for ensuring the data did not identify individual communities or people and that the interpretation of the results was consistent with community values.

\section{RESULTS}

For the period 1 January 2007 to 31 December 2009 there were a total of 234 IPD notifications in this regional area of NSW. Initially 168 (72\%) notifications had Aboriginal and Torres Strait Islander status recorded in the notifiable conditions database. After referring to the routine hospital admission data, the status recorded increased to 232 (99\%).

Referring to the accessible routine hospital admission data for the 66 notifications in the 5-49 years age group required two hours of work for a Surveillance Officer. Prospective data checking during 2009-2010 confirmed that it takes less than five minutes to check and update the notification when there is easy access and approvals in place for data checking.

Of the 234 notifications of IPD in residents of this regional area, 12 were recorded as Aboriginal people, and there were no patients who identified as Torres Strait Islanders in their hospital admission (Table 1). All of the 
Table 1. Number of IPD notifications in the regional area of New South Wales, by Aboriginal and Torres Strait Islander status, 2007-2009

\begin{tabular}{|c|c|c|c|c|c|c|}
\hline Age Group & $\begin{array}{c}\text { Aboriginal and } \\
\text { Islander }\end{array}$ & $\begin{array}{l}\text { Torres Strait } \\
\text { (\%) }\end{array}$ & Non-Indigenous & Unknown & $\begin{array}{l}\text { Unknown prior to } \\
\text { data checking }\end{array}$ & Total \\
\hline $0-4$ years & 5 & $19 \%$ & 22 & 0 & 0 & 27 \\
\hline $5-49$ years & 3 & $5 \%$ & 62 & 1 & 65 & 66 \\
\hline $50+$ years & 4 & $2 \%$ & 136 & 1 & 1 & 141 \\
\hline Total & 12 & $5 \%$ & 220 & 2 & 66 & 234 \\
\hline
\end{tabular}

notifications in the $5-49$ years age group had Aboriginal and Torres Strait Islander status recorded as "unknown" before the data checking was conducted.

The crude notification rate for IPD in non-Aboriginal people over the study period was 8.9 per 100000 population, while for Aboriginal and Torres Strait Islander people the rate was 12.2 per 100000 population, though not significantly different (Table 2).

After direct age-standardization, the relative risk (RR) was significantly higher for Aboriginal people aged $0-4$ years of age (RR 2.68, 1.02-7.09 95\% Cl). The rates of disease in the age groups 5-49 years and 50 years and older were not different (Table 2).

Aboriginal and Torres Strait Islander children aged 0-4 years of age had a statistically significant higher relative risk of being notified with IPD. Other age groups did not have a significantly higher relative risk.

\section{DISCUSSION}

Surveillance of vaccine-preventable diseases is important to allow targeted vaccine strategies where necessary and to inform evaluations of existing vaccination programmes.
Accessing Aboriginal and Torres Strait Islander status by referring to routine hospital admission data for the 66 IPD cases in the 5-49 year age group and updating the notification data required only two hours in total to complete. Time constraints at a public health unit level are a limiting factor for completeness of data, but where there is easy and approved access for data checking this should be undertaken. As a result this regional area of NSW can now report Aboriginal status for nearly all notified IPD cases from the period 2007-2009. This information will be updated annually and allows the Hunter New England Aboriginal Health Partnership to plan and evaluate services to Aboriginal communities.

The method used to collect Aboriginal and Torres Strait Islander status for admissions with IPD could also be used with other notifiable conditions that result in hospital admission such as invasive meningococcal disease. The surveillance method could be applied in other jurisdictions and settings where electronic access to hospital admission data for public health units is available and approved. Not only will this provide a more complete epidemiological profile but the surveillance can also improve the public health response and enable more culturally appropriate actions to be taken.

Table 2. IPD notification rates in residents of the regional area of New South Wales, standardized by age group and Aboriginal and Torres Strait Islander status with relative risk of IPD in Aboriginal and Torres Strait Islander populations, 2007-2009

\begin{tabular}{|c|c|c|c|c|c|}
\hline $\begin{array}{c}\text { Population by age } \\
\text { group }\end{array}$ & Notifications & Population & $\begin{array}{l}\text { Notification ratel } \\
100000 \text { population }\end{array}$ & RR & $\begin{array}{l}\text { 95\% Confidence } \\
\text { Interval }\end{array}$ \\
\hline \multicolumn{6}{|l|}{ Non-indigenous } \\
\hline $0-4$ years & 22 & 148344 & 14.83 & & \\
\hline 5-49 years & 62 & 1431947 & 4.33 & & \\
\hline $50+$ years & 136 & 898607 & 15.13 & & \\
\hline Total & 220 & 2478898 & 8.87 & & \\
\hline \multicolumn{6}{|c|}{ Aboriginal and Torres Strait Islander } \\
\hline 0-4 years & 5 & 12559 & 39.81 & 2.68 & 1.02 to 7.09 \\
\hline 5-49 years & 3 & 74080 & 4.05 & 0.94 & 0.29 to 2.98 \\
\hline $50+$ years & 4 & 11968 & 33.42 & 2.21 & 0.82 to 5.97 \\
\hline Total & 12 & 98607 & 12.17 & 1.37 & 0.77 to 2.45 \\
\hline
\end{tabular}


The notification rate in non-Aboriginal people in the regional area, 8.9 per 100000 population, is similar to the rate reported for all NSW residents, 8.3 per 100000 population in $2006 .^{3}$ The reported rate using the complete data for notified IPD in Aboriginal and Torres Strait Islander populations in this regional area of NSW was 12.2 per 100000 population, which was lower than that reported for Australia (28.0 per 100000 population in 2006).

Although the rate of IPD in the 5-49 years age group was similar in Aboriginal and Torres Strait Islander and non-Aboriginal in the study populations, monitoring these data over time will enable a better understanding of the importance of this disease in the community. ${ }^{3}$

Several limitations to this study mean that the results need to be treated with caution. Relatively few notifications were received during the study period resulting in wide confidence intervals, although the increased risk in children under 5 years was statistically significant. A further limitation may be that even though it is policy of NSW Health that all people admitted to hospital are asked about their Aboriginal and Torres Strait Islander status, ${ }^{7}$ it is possible that a small number of Aboriginal and Torres Strait Islander people with IPD may not have been identified in the routine hospital admission data. Levels of Aboriginal and Torres Strait Islander identification in NSW have improved with current identification at $88 \% .^{8}$ Hospital identification levels at $88 \%$ may not be sufficiently high for the results to fully represent the population. It is also recognized that notifications of IPD can be an underestimate of the burden of disease in a population.

Controlling for socioeconomic status is not feasible with the notification data available in NSW as there is no routine collection of a notified individual's socioeconomic status. The small numbers of notifications also do not support an ecological analysis.

\section{CONCLUSIONS}

Referring to routine hospital admission data is a useful and time-efficient surveillance strategy to increase the proportion of IPD notifications with Aboriginal and Torres Strait Islander status. This surveillance method may also be useful in other important notifiable diseases where people are admitted to hospital.

Including Aboriginal and Torres Strait Islander status in the surveillance of IPD is important to enable the detection of changes in the epidemiology of the disease and to inform strategies for further reducing the impact of this serious illness.

Aboriginal and Torres Strait Islander children aged 0-4 years have a two- to threefold higher rate of invasive pneumococcal disease than non-Aboriginal children and thus high levels of timely pneumococcal immunization coverage remain important for young Aboriginal and Torres Strait Islander children.

\section{Conflict of interest}

None declared.

\section{Funding}

None.

\section{Acknowledgements}

The authors thank Fakhrul Islam, Hunter New England Population Health, for valuable assistance in reporting of population and notification data.

\section{References:}

1. Musher DM. Strepococcus pneumoniae. In: Mandell GL, Bennett JE, Dolin R, editors. Mandell, Douglas, and Bennett's Principles and Practice of Infectious Diseases, 7th edition. Philadelphia, Churchill Livingstone Elsevier, 2009, p 2623-2642.

2. Heymann DL, editor. Control of Communicable Diseases Manual 19th Edition. Washington DC, American Public Health Association, 2008.

3. Roche PW et al. Enhanced Invasive Pneumococcal Disease Surveillance Working Group; Pneumococcal Working Party of the Communicable Diseases Network Australia. Invasive pneumococcal disease in Australia, 2006. Communicable Diseases Intelligence, 2008, 32:18-30. pmid:18522302

4. The Health and Welfare of Australia's Aboriginal and Torres Strait Islander Peoples 2008. Australia, Bureau of Statistics and Institute of Health and Welfare, 2008 (http://www. aihw.gov.au/publications/index.cfm/title/10583, accessed on 7 June 2011)

5. Thomson $\mathrm{N}$ et al. Overview of Australian Indigenous health status, December 2009. Australian Indigenous HealthInfoNet, 2009 (http://www.healthinfonet.ecu.edu.au/health-facts/overviews, accessed on 7 June 2011).

6. Menzies $\mathrm{R}$ et al. Vaccine preventable diseases and vaccination coverage in Aboriginal and Torres Strait Islander people, Australia 2003 to 2006. Communicable Diseases Intelligence, 2008, 32 Suppl:S2-67. pmid:18711998

7. Policy Directive PD. 2005547 Aboriginal and Torres Strait Islander Origin - Recording of Information of Patients and Clients. New South Wales, Department of Health: Aboriginal Health, 2005 (http://www.health.nsw.gov.au/policies/pd/2005/ pdf/PD2005_547.pdf, accessed on 7 June 2011).

8. Health Services Series no. 35: Indigenous identification in hospital separations data-quality report. Canberra, Australian Institute of Health and Welfare, 2010 (http://www.aihw.gov.au/ WorkArea/Download Asset.aspx?id=6442457092, accessed on 7 June 2011). 